\title{
Matrix Moment Methods in Perturbation Theory, Boson Quantum Field Models, and Anharmonic Oscillators
}

\author{
S. Graffi and V. Grecchi \\ Istituto Nazionale di Fisica Nucleare, Sezione di Bologna, Bologna, Italy
}

Received June 1, 1973

\begin{abstract}
A matrix moment problem is considered in connection with any $x^{2 m}(m=2,3,4, \ldots)$ anharmonic oscillator as well as the $\left(: \phi^{2 m}(x): g(x)\right)_{2}(m=2,3)$ field theory models, whose Rayleigh-Schrödinger perturbation expansions for the ground state eigenvalue are known to diverge. The approximants related to such a problem are proven to converge to the eigenvalue, when applied to an expansion of the Brillouin-Wigner type. These approximants, whose construction involves only matrix elements occurring in the Rayleigh-Schrödinger expansion, are the approximants of a $J$-type matrix continued fraction, i.e. the $[N-1, N]$ matrix Padé approximants. The explicit analytical expression of matrix continued fraction is found in the anharmonic oscillators case.
\end{abstract}

\section{Introduction}

The approximation problem for the ground state eigenvalue and eigenvector of a class of perturbation problems has been recently treated by McClary [1]. This class includes the $g\left(: \phi^{2 m}(x): g(x)\right)_{2}(m=2,3)$ field theory models as well as the $x^{2 m}(m=2,3)$ anharmonic oscillators. In both cases the Rayleigh-Schrödinger (R-S) perturbation expansion for the ground state eigenvalue $E(g)$ is known to be divergent as fast as $((m-1) n)$ !, this last result being valid for arbitrary finite $m$ [2]. For any model in the class mentioned above but the $\left(: \phi^{6}(x): g(x)\right)_{2}$ it has been proven that the divergent R-S perturbation expansion is Borel summable to the exact solution $[3,4]$; in addition, for the $m=2,3$ anharmonic oscillators the expansion is also Stieltjes summable [5]. This last result provides a strong approximation statement, since the Stieltjes method is equivalent to the convergence of the Method of Moments (i.e. Padé) approximants.

No rigorous approximation statement directly generated by a summation method was however known for any field theoretical model, nor for the $x^{2 m}$ anharmonic oscillators with $m>3$.

Now it has been shown in Ref. [1] that a monotonic sequence of approximants converging to $E(g)$ for $g$ real and positive may be obtained, 
for any model belonging to the class defined above, through the Method of Moments approximants applied to an expansion of the BrillouinWigner type.

The convergence proof rests on a characterization [6] of semibounded self-adjoint operators in Hilbert space obtained, through the inclusion of the power moment problem into the theory of self-adjoint operators with simple spectrum (7), from the Carleman determination criterion for the Stieltjes moment problem.

This method, as it will clearly appear later on, does not allow a direct generalization of the result to any $\left(: \phi^{2 m}(x): g(x)\right)_{2}$ field theory model nor to any $x^{2 m}$ anharmonic oscillators, $m>3$.

Purpose of this paper is to show that, if an approximation scheme of essentially the same nature is considered, i.e. the Matrix Method of Moments approximants, it is possible to obtain a convergence proof valid not only for the class of models treated by McClary, but also for any anharmonic oscillator $x^{2 m}, m=2,3,4 \ldots$.

In complete analogy with (1), for the field theoretical models the proof rests on the above mentioned self-adjointness criterion and, as it will be seen later on, the extension to any one-dimensional anharmonic oscillator is possible because in this last case the vectors occurring in the R-S expansion needed to build the matrix approximants span the whole Hilbert space. This fact, in addition, enables us to get an explicit expression of all the coefficients of the matrix continued fraction which generates the approximants.

Throughout the present paper we will closely follow the presentation and notation of (1), beginning in the next section with the definition of our problem and the list of the known properties needed in the subsequent discussion. We shall also make explicit use of some results proven in (1) in Section III, where we prove the validity conditions of our approximation statement. In Section IV the matrix approximants will be shortly reviewed, and in Section V they will be applied to the problems under discussion.

\section{Class of Problems under Consideration}

Our considerations apply to the perturbed Hamiltonian

where

$$
H_{0}+g V, g \geqq 0
$$

i) in the $\left(: \phi^{2 m}(x): h(x)\right)_{2} m=2,3$ models $\phi(x)$ is a relativistic quantum field in a two dimensional space-time, with $H_{0}=\int a^{+}(k) a(k)\left(k_{0}^{2}+m^{2}\right)^{1 / 2} d k$ and $V=\int: \phi^{2 m}(x): h(x) d x, m=2,3, \ldots$ is an interaction space cut-off by a smooth function $h(x)$ equal to 1 for small $|x|$ and to 0 for large $|x|$. 
ii) in the quantum mechanical anharmonic oscillator case $H_{0}=\frac{1}{2}\left(\frac{d^{2}}{d x^{2}}+x^{2}-1\right)$, and

$$
V=2^{-m}((2 m-1) !)^{-1 / 2} H_{2 m}(x), \quad m=2,3,4, \ldots .
$$

The following statements hold in both cases (see Glimm and Jaffe [9] and Höegh-Krohn and Simon [10] for the: $\phi^{2 m}(x)$ : models; Simon [11] for the anharmonic oscillators):

A) $H_{0}$ is a self-adjoint operator in a Hilbert space $F$ (the Fock space for the : $\phi^{2 m}(x): h(x)$ models, $L^{2}(-\infty,+\infty)$ for the anharmonic oscillators). It has non-degenerate isolated least (ground state) eigenvalue 0 , and eigenvector $\Omega . V$ is a symmetric operator, and $(\Omega, V \Omega)=0 . H_{0}+g V$ is a self-adjoint operator with domain $D(H)=D\left(H_{0}\right) \cap D(V)$; it has a ground state eigenvalue $E(g)$ with eigenvector $\Psi(g)$.

$$
\begin{gathered}
H_{0} \Omega=0 \\
\left(H_{0}+g V-E(g)\right) \Psi(g)=0 .
\end{gathered}
$$

We assume the normalization $\|\Omega\|=1, \Psi(g)=\Omega+\Psi^{\perp}(g)$, with

$$
\left(\Omega, \Psi^{\perp}(g)\right)=0 .
$$

B) A "number" operator $N$ may be defined as a self-adjoint operator in $F$, with eigenvalues $0,1, \ldots$ and eigenvectors $\psi_{n}$, called $n$-particle vectors. For the anharmonic osciljtors, $N=H_{0}$ and the $\psi_{n}$ are the harmonic oscillator eigenstates. $D(V)$ contains all $n$-particle vectors. Let now $F^{+}, F^{-}$be the Hilbert spaces defined by: $F^{+}=\bigoplus_{n=1}^{\infty} F_{2 n}$, $F^{-}=\bigoplus_{n=1}^{\infty} F_{2 n-1}$, where $F_{n}$ is the $n$-particle subspace.

We have: $F=F^{+} \oplus F^{-} \oplus \Omega=F^{\perp} \oplus \Omega$, with $F^{\perp}=F^{+} \oplus F^{-}$. If $P^{\perp}$ is the orthogonal projection onto $F^{\perp}, P^{+}$onto $F^{+}$, and $P^{-}$onto $F^{-}$, and $A$ an operator in $F$, let $A^{\perp}, A^{+}, A^{-}$the operators in $F^{\perp}, F^{+}, F^{-}$defined by $P^{\perp} A P^{\perp}, P^{+} A P^{+}, P^{-} A P^{-}$, respectively. We have:

C) $\left(H_{0}+g V\right)^{\perp}$ is a self-adjoint operator in $F^{\perp}$ [1]. Since $F^{+}, F^{-}$are left invariant by $\left(H_{0}+g V\right)^{\perp}=H^{\perp}$, it follows that $H^{+}=P^{+}\left(H_{0}+g V\right) P^{+}$ and $H^{-}=P^{-}\left(H_{0}+g V\right) P^{-}$are self-adjoint operators in $F^{+}, F^{-}$, respectively. $\mathrm{H}_{0}^{+}$is a strictly positive self-adjoint operator in $F^{+}$, possessing therefore a self-adjoint square root, a bounded self-adjoint inverse and a bounded self-adjoint inverse square-root, denoted respectively by $h^{1 / 2}, h^{-1}, h^{-1 / 2}$.

D) $H_{0}+g V$ is essentially self-adjoint on the domain of $n$-particle vectors in $D\left(H_{0}\right)$. As in C), it follows that $\left(H_{0}+g V\right)^{+},\left(H_{0}+g V\right)^{-}$, 
$\left(H_{0}+g V\right)^{\perp}$ are essentially self-adjoint on the domains $D^{+}, D^{-}, D^{\perp}$ of respectively, $2 n, 2 n-1, n(n=1, \ldots)$ particle vectors in $D\left(H_{0}\right) \cap F^{+}$, $D\left(H_{0}\right) \cap F^{-}, D\left(H_{0}\right) \cap F^{\perp}$. The sets of vectors $h^{1 / 2} D^{+}, h^{1 / 2} D^{-}, h^{1 / 2} D^{+}$are dense in $F^{\perp}, F^{-}, F^{+}$respectively.

\section{Essential Self-Adjointness, Finite Multiplicity, and Matrix Moment Problem}

Let us proceed as in Ref. [1] in order to derive an implicit equation of the Brillouin-Wigner (B-W) type for the eigenvalue $E(g)$.

We write:

where:

$$
\Psi^{\perp}(g)=\Psi^{+}(g)+\Psi^{-}(g)
$$

$$
\begin{gathered}
\Psi^{+}(g) \in F^{+} ; \Psi-(g) \in F^{-} \quad \text { and of course }\left(\Psi^{+}, \Psi^{-}\right)=0 \\
\left(\Psi^{+}, \Omega\right)=0 ; \quad\left(\Psi^{-}, \Omega\right)=0 .
\end{gathered}
$$

Taking in (1.1) the scalar product with $\Omega$ and applying $P^{+}$we get the following system of two equations for $E(g)$ and $\Psi^{+}(g):\left(V \Omega \in F^{+}\right)$

$$
\begin{gathered}
{\left[\left(H_{0}+g V\right)^{+}-E(g)\right] \Psi^{+}(g)+g V \Omega=0} \\
E(g)=\left(g V \Omega, \Psi^{+}(g)\right) .
\end{gathered}
$$

By elimination of $\Psi^{+}(g)$ we get:

where:

$$
E(g)=f(E(g), g)
$$

$$
f(E(g), g)=-\left(g V \Omega,\left[\left(H_{0}+g V\right)^{+}-E\right]^{-1} g V \Omega\right) .
$$

Now exactly as in (1) one proves that the self-adjoint operator $\left(H_{0}+g V\right)^{+}$has lower bound $G(g)>E(g)$. This implies that $f(E, g)$ exists as a negative, monotonically decreasing function of $E$ in the interval $-\infty<E<G(g)$, so that the eigenvalue $E(g)$ is the only solution of (3.3) in this interval.

To prove our approximation statement we need the following results:

Theorem 1. Let the operator $A$ in $F$ be defined as:

$$
A=h^{-1 / 2}(g V-E)^{+} h^{-1 / 2} .
$$

The there is a $C(g)>E(g)$ such that if $E<C$ then:

(i) $A+1$ is a strictly positive operator, and

(ii) $A$ is essentially self-adjoint on any dense domain $D$ of $2 n$-particle vectors. 
Note. This theorem is the generalization of Theorem II of Ref. [1], to which the reader is referred for the proof of (i), and of (ii) for the field theoretical case. We provide an alternative proof of (ii) valid for any $m$ in the quantum mechanical case, since that of (1) rests on the selfadjointness criterion applicable only for $m=2,3$. If, indeed, the symmetric operator $A$ satisfies condition (i), its essential self-adjointness is ensured when it has a dense set of Stieltjes vectors [6]. (A Stieltjes vector for an operator $B$ is any vector $\phi \in \bigcap_{n=1}^{\infty} D\left(B^{n}\right)=C^{\infty}(B)$, such that

$$
\left.\sum_{n=0}^{\infty}\left\|B^{n} \phi\right\|^{-\frac{1}{2 n}}=\infty\right) .
$$

Now, $\chi$ being any finite particle vector, (i.e. a finite sum of $n$-particle vectors) there exists a $d$ such that

$$
\left\|\left(h^{-1 / 2} V h^{-1 / 2}\right)^{n} \chi\right\| \leqq(d n)^{(m-1) n}
$$

for any $x^{2 m}$ as well as any $\left(: \phi^{2 m}(x): h(x)\right)_{2}$ model $[1,10,11]$.

It follows that the identification of the $2 n$-particle vectors as Stieltjes ones for $A$ is possible only for $m=2,3$.

Proof of (ii). Since (i) holds, and $A+1$ is trivially symmetric, to prove its essential self-adjointness we need only to show that its range $R(A+1)$ is dense in $F^{+}$.

Suppose there exists a vector $\phi \in F^{+}$orthogonal to $R(A+1)$

$$
(\phi,(A+1) \psi)=0, \quad \psi \in D .
$$

Since $h^{-1 / 2}$ is a bounded, self-adjoint and strictly positive operator in $F^{+}$it is easily seen that the above orthogonality is equivalent to

$$
\left(h^{-1 / 2} \phi,\left[\left(H_{0}+g V\right)^{+}-E\right] h^{-1 / 2} \psi\right)=0 .
$$

Since, by C), $\left(H_{0}+g V\right)^{+}$is essentially self-adjoint on $D$ with lower bound $G>E$ [1], and any element of $D$ is eigenvector of $h^{-1 / 2}$, we must have $h^{-1 / 2} \phi=0$, i.e. $\phi=0$.

Thus $A+1$, and hence $A$, is essentially self-adjoint. Consider again the quantum mechanical case, $V=2^{-m}\left((2 m-1 !)^{-1 / 2} H_{2 m}(x)\right)$, and let $A$ be defined as above. We have:

Theorem 2. The multiplicity of the spectrum of $A$ is not greater than $m$.

Proof. By the well known characterization of self-adjoint operators with spectrum of finite multiplicity [8], it is enough to identify in $D(A) m$ linearly independent vectors $g_{i}$ ( $m$-dimensional generating basis) such that the set of vectors $E(\Delta) g_{i}, i=1, \ldots m$, is dense in $F^{+}$. Here $E(t)$ stands 
for the resolution of the identity corresponding to $\bar{A}=A^{*}$, and $\Delta$ runs through the set of all intervals on $[-1, \infty]$.

Let now $g_{i}=\left(\pi^{1 / 2}(2 i) !\right)^{-1 / 2} 2^{-i} e^{-\frac{x^{2}}{2}} H_{2 i}(x), i=1, \ldots m$.

As it is proven in Appendix, an orthogonalization procedure of the Gram-Schmidt type applied to the sequence of linearly independent vectors $A^{n} g_{i}, n=0,1, \ldots ; i=1, \ldots m$; yields the sequence of the normalized harmonic oscillator eigenstates $e_{2 m}, m=1,2, \ldots$, i.e. the set of vectors $A^{n} g_{i} ; n=0,1 \ldots ; i=1, \ldots m$ is dense in $F^{+}$. This implies that the set $E(\Delta) g_{i}, i=1, \ldots m$, is also dense, since the integral representation:

$$
\left(A^{n} g_{i}, h\right)=\int_{-1+\varepsilon}^{\infty} t^{n} d\left(E(t) g_{i}, h\right), \quad i=1, \ldots m
$$

clearly shows the non existence of a vector $h \neq 0$ orthogonal to $E(t) g_{i}$, for any $t$ and any $i=1, \ldots m$. Given the $m$-dimensional generating basis $g_{i}, i=1, \ldots m$ defined above, let us introduce the following $m \times m$ Hermitian matrix:

$$
M=M_{i, k}=\left(g_{i},(1+A)^{-1} g_{k}\right)
$$

We have:

$$
f(E, g)=-g^{2} M_{m, m}=-g^{2}\left(h^{-1 / 2} V \Omega,(1+A)^{-1} h_{-}^{-1 / 2} V \Omega\right) .
$$

For the proof see (1).

With $E(t)$ as above, we can put the matrix $M$ under the form of a matrix Stieltjes transform [13]:

$$
M=\int_{-1+\varepsilon}^{\infty} \frac{d T(t)}{1+t}
$$

where the matrix distribution function $T(t)$ is defined by:

$$
T(t) \equiv T_{i k}(t)=\left(g_{i}, E(t) g_{k}\right), \quad i, k=1, \ldots m,
$$

its moments being of course the $m \times m$ matrices:

$$
m_{n}^{i, k}=\int_{-1+\varepsilon}^{\infty} t^{n} d T(t)=-\left(g_{i}, A^{n} g_{k}\right), \quad i, k=1, \ldots m
$$

We recall that a matrix distribution function $T(t)$ is a Hermitian matrix function $T(t)=T_{i k}(t)$ such that:

1) $\xi_{k}$ being arbitrary complex numbers, the quadratic forms:

$\sum_{i, k=1}^{m}\left[T_{i k}\left(t^{\prime \prime}\right)-T_{i k}\left(t^{\prime}\right)\right] \xi_{i} \bar{\xi}_{k}$ are semidefinite positive when $t^{\prime \prime}>t^{\prime}$;

2) $T(-\infty)=0$ (the null matrix). $T(t-0)=T(t)$. 
Formula (3.7) defines a matrix moment problem of the Hamburger type. The uniqueness of the matrix distribution function (i.e. the determination of the matrix moment problem) follows from a result of Krein [14], which allows the extension to the matrix case of a well-known result of Stone [7] valid for the classical Hamburger moment problem.

Theorem 3. Let $A$ be a self-adjoint operator in a Hilbert space $H$ with multiplicity not greater than $m, E(t)$ its resolution of the identity, and $g_{1} \ldots g_{m}$ a m-dimensional generating basis belonging to $C^{\infty}(A)$ such that the linear hull of the vectors $A^{n} g_{i}, i=1, \ldots m, n=0,1,2, \ldots$ is a core for $A$.

Then the matrix sequence:

$$
S_{n}=\left(g_{i}, A^{n} g_{k}\right)=\int_{-1+\varepsilon}^{\infty} t^{n} d T(t)
$$

$T(t)$ being the matrix distribution function $T(t)=\left(g_{i}, E(t) g_{k}\right)$, is a determined Hamburger moment sequence.

Proof. Let us apply the orthogonalization procedure described in Appendix to the sequence of vectors $g_{i} \ldots g_{m}, A g_{1} \ldots A g_{m}, \ldots$ $A^{n} g_{1} \ldots A^{n} g_{m} \ldots$. We end up with a sequence of orthonormal vectors $e_{k}$, $k=1,2, \ldots$, satisfying a $2 m+1$-term recurrence relation:

$A e_{k}=b_{k-m} e_{k-m}+a_{k, k-m+1} e_{k-m+1}+\cdots+a_{k, k} e_{k}+\cdots+b_{k} e_{k+m}, \quad k=1,2 \ldots$

where

$$
a_{k, j}=\bar{a}_{j, k} ; b_{k}>0, j, k>0 ; a_{k, j}=b_{k}=0, j, k \leqq 0 .
$$

If we arrange the orthonormal set $e_{k}, k=0,1, \ldots$ into $m$-component column vectors $E_{k}$ defined in the following way:

$$
E_{k}=\left(\begin{array}{c}
e_{k m+1} \\
\vdots \\
e_{k m+m}
\end{array}\right)
$$

it is easily seen that operator $A$ is defined on the unit column vectors $E_{k}$ by a three-term matrix recurrence relation:

$$
A E_{k}=A_{k, k-1} E_{k-1}+A_{k, k} E_{k}+A_{k, k+1}, \quad k=0,1 \ldots
$$

where:

$$
\begin{aligned}
& A_{k, k}=A_{k, k}^{+}=\left(\begin{array}{ccc}
a_{k m+1, k m+1} & \ldots & a_{k m+1, k m+m} \\
a_{k m+m, k m+1} & \ldots & a_{k m+m, k m+m}
\end{array}\right),
\end{aligned}
$$

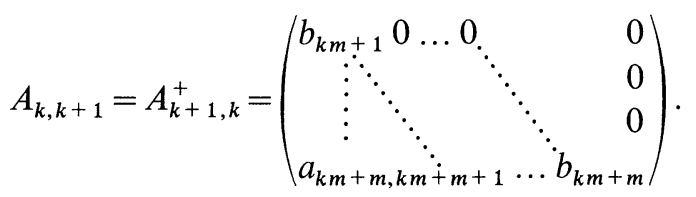


It follows that on the basis $e_{k}, k=0,1, \ldots$ the matrix of the operator $A$ is a $J_{m}$ one having the form:

$$
J_{m}=\left(\begin{array}{llll}
A_{0,0} & A_{0,1} & 0 & 0 \\
A_{1,0} & A_{1,1} & A_{1,2} & 0 \\
0 & A_{2,1} & A_{2,2} &
\end{array}\right),
$$

i.e. a $J_{m}$ matrix, generalization of the ordinary Jacobi one, is an infinite Hermitian matrix having the form $\left\|A_{i, k}\right\|, i, k=0,1,2 \ldots$, where $A_{i, k}$ are $m$-matrices (i.e. square matrices with $m$ rows and $m$ columns), with $A_{i, i \pm 1}$ non singular and $A_{i, k}=0$ for $|i-k|>1$.

Since the set of vectors $e_{k}$ is a core for $A$, the minimal closed operator generated by $J_{m}$ on the basis $e_{k}$ coincides with the self-adjoint operator $A$, i.e. $A$ is represented by the matrix $J_{m}$ : hence the corresponding operator in $1^{2}$ generated by the infinite matrix $J_{m}$ is self-adjoint. The theorem is then proved by a direct application of the Krein result [14], which states that $T(t)=\left(g_{i}, E(t) g_{k}\right), i, k=1, \ldots m$ is the unique normed solution of the moment problem (3.8) if and only if the deficiency indices of the operator generated by $J_{m}$ in $1^{2}$ are $(0,0)$.

If we now indicate with $\bar{A}=A^{*}$ the closure of the essentially selfadjoint operator $A$ defined by (3.5), Theorems 1 and 2 show that the conditions of Theorem 3 are satisfied as far as the matrix moment problem (3.7) for the quantum mechanical case is concerned: this is because, as it is shown in Appendix, in the present case the basis $e_{n}$ turns out to coincide with that of the harmonic oscillator eigenstates, which is by Theorem 1 a core for $A$. The above procedure does not carry over directly to the field theoretical models, because it is clearly impossible in that case to find a finite dimensional generating basis. However, if we limit ourselves as in (1) to the models $\left(: \phi^{2 m}(x): h(x)\right)_{2}, m=2,3$, it is possible to obtain analogous results through the above mentioned essential self-adjointness criterion. Let now $V=g \int: \phi^{2 m}(x): h(x) d x$, $m=2,3$, and $A$ be defined by (3.5).

By Theorem 2, $A$ is essentially self-adjoint on any dense domain of $2 n$-particle vectors. Let $E(t)$ the resolution of the identity corresponding to $\bar{A}$, and consider the following $m$ linearly independent and orthogonal vectors of $C^{\infty}(A)$ :

$$
g_{m}=c_{m} h^{-1 / 2} V \Omega ; \quad g_{i}=c_{i} h^{-1 / 2} f_{2 i}, \quad i=1, \ldots m-1,\left\|g_{i}\right\|=1
$$

where $f_{2 i}$ is the $2 i$-particle vector defined by: $V h^{-1} V \Omega=\sum_{i=1}^{2 m} f_{2 i}$. As before, let us define the matrix:

$M=\left(g_{i},(1+A)^{-2} g_{k}\right) \quad i, k=1, \ldots m$; and the matrix distribution function $T(t)$ through: $\left(g_{i}, E(t) g_{k}\right)=T(t) ; i, k=1, \ldots m$. 
We have again $g^{2} M_{m, m}=-\left\|h^{-1 / 2} V \Omega\right\|^{-2} f(E, g)$, and:

Theorem 4. The sequence:

$$
m_{n}=\int_{-1+\varepsilon}^{\infty} t^{n} d T(t)=\left(g_{i}, A^{n} g_{k}\right), \quad i, k=1, \ldots m
$$

is a determined matrix moment sequence.

Proof. Consider the vectors $A^{n} g_{i}, n=0,1,2 \ldots ; i=1, \ldots m$. Let $H_{G}$ the Hilbert space spanned by these vectors and let $B$ the restriction of $A$ to $H_{G}$. As a restriction of the essentially self-adjoint operator $A, B$ will be a symmetric operator in $H_{G}$, with $B+1$ strictly positive. The bound (3.6) shows that any finite particle vector is a Stieltjes vector for $A$.

Since $V$ connects only vectors whose particle number differs by no more than six, any vector $A^{n} g_{i}, n=0,1, \ldots ; i=1, \ldots m$ is a finite particle and thus a Stieltjes one; hence $B$ has a dense set of Stieltjes vectors and by the above mentioned criterion [6] is essentially selfadjoint on the domain $A^{n} g_{i}, i=1, \ldots m ; n=0,1,2, \ldots$ in $H_{G}$. Our assertion is thus proved applying Theorem 3 to $\bar{B}$.

It is now possible, for the class of problems under consideration, to approximate the matrix $M$ and hence $f(E, g)$ through the method to be briefly described in the next section, which consists in the generalization to the matrix case of the classical theory of Jacobi matrices, orthogonal polynomials, and continued fractions.

\section{Matrix Orthogonal Polynomials and Approximants}

Let $S_{n}$ be a sequence of hermitian $m$-matrices with complex elements and $T(x)$ a matrix distribution function, solution (not necessarily unique) of the following Hamburger matrix moment problem:

$$
S_{n}=\int_{-\infty}^{+\infty} x^{n} d T(x)
$$

Consider the class $C_{m}$ of all matrix polynomials whose coefficients are $m$-matrices. Given two polynomials $P(x)=\sum_{k=0}^{n} c_{k} x^{k}, Q(x)=\sum_{k=0}^{m} d_{k} x^{k}$ belonging to $C_{m}$, let us define a matrix "scalar product" $(P, Q)$ through the following properties:

i) $(P+Q, R)=(P, R)+(Q, R)$

ii) $(C P, Q)=C(P, Q),(P, C Q)=(P, Q) C^{+}$where $C$ is a $m$-matrix independent of $x$;

iii) $\left(I x^{i}, I x^{j}\right)=S_{i+j}$ where $I$ is the unit $m$-matrix. 
It is easy to see that the above defined "scalar product" may be written under the form:

$$
(P, Q)=\int_{-\infty}^{+\infty} P(x) d T(x) Q^{+}(x) .
$$

Hence it follows that

$$
(P, P)=\int_{-\infty}^{+\infty} P(x) d T(x) P^{+}(x)=\sum_{i, k=1}^{n} c_{i} S_{i+k} c_{k}^{+}
$$

is a positive definite $m$-matrix.

It is therefore possible to define a sequence of matrix polynomials $P_{k}(x)$ of degree $k$, orthonormal with respect to the scalar product defined above, i.e. $\left(P_{k}, P_{j}\right)=\delta_{k j} I$, and satisfying a three-recurrence relation:

$$
x P_{k}=A_{k, k-1} P_{k-1}+A_{k, k} P_{k}+A_{k, k+1} P_{k+1}, \quad k=1,2 \ldots
$$

where:

$$
A_{k, k+1}=A_{k+1, k}^{+}=\left(x P_{k}, P_{k+1}\right), \quad A_{k, k}=A_{k, k}^{+}=\left(x P_{k}, P_{k}\right), \quad k=0,1,2, \ldots
$$

$A_{k, k+1}$ being non-singular $m$-matrices.

For the case of a moment problem generated by a self-adjoint operator they coincide with the coefficients of the $J_{m}$ matrix of the operator itself (see Appendix).

The recurrence relations (4.3), together with the initial conditions $P_{0}=I, P_{1}=A_{0,1}^{-1}\left(x-A_{0,0}\right)$, yield an equivalent definition of the $P_{k}$, and with the initial conditions $Q_{0}=0, Q_{1}=A_{0,1}^{-1}$ give rise to a sequence of polynomials $Q_{k}$, of degree $k-1$, which are easily verified to satisfy the relation:

$$
Q_{k}(y)=\left(\frac{P_{k}(x)-P_{k}(y)}{x-y}, I\right) .
$$

It is convenient to introduce also the matrix polynomials $Y_{k}(x)$, $Z_{k}(x)$, defined in the following way:

$$
P_{k}(x)=C_{k} Y_{k}(x) ; Q_{k}(x)=C_{k} Z_{k(x)}, \quad k=0,1,2 \ldots
$$

where

$$
\begin{aligned}
& C_{k}=A_{k-1, k}^{-1} A_{k-1, k-2} A_{k-3, k-2}^{-1} \ldots A_{1,0}, \quad k \text { even } \\
& C_{k}=A_{k-1, k}^{-1} A_{k-1, k-2} A_{k-3, k-2}^{-1} \ldots A_{0,1}^{-1}, \quad k \text { odd } ; C_{0}=I .
\end{aligned}
$$

For these polynomials we get then the recurrence relations:

$$
\begin{aligned}
Y_{n+1}(x) & =\left(x A_{n}+B_{n}\right) Z_{n}(x)-Z_{n-1}(x), \quad n=1,2, \ldots \\
Z_{n+1}(x) & =\left(x A_{n}+B_{n}\right) Z_{n}(x)-Z_{n-1}(x), \quad n=1,2, \ldots \\
Y_{0} & =I, Y_{1}=x A_{0}+B_{0} ; \quad Z_{0}=0, Z_{1}=I .
\end{aligned}
$$


where:

$A_{n}=A_{n}^{+}=C_{n}^{+} C_{n} \quad$ is positive definite, and $B_{n}=B_{n}^{+}=-C_{n}^{+} A_{n, n} C_{n}$.

Suppose now that the integrations in (4.1) are bounded from below by $-M, M>0$, i.e. $T(x)$ constant for $x<-M$ :

$$
S_{n}=\int_{-M}^{\infty} x^{n} d T(x)
$$

From the orthogonality of the matrix polynomials $P_{k}(x)$ it follow that the poles of $P_{k}^{-1}(x)$ are simple and lie all inside the interval $[-M, \infty]$. In addition, the coefficient of the highest power in $P_{k}(x)$ is positive definite, as it is implied by the recurrence relations with the given initial conditions. We can thus conclude that $(-1)^{k} P_{K}(x)$, as well as $(-1)^{k} Y_{k}(x)$, is positive definite for $x<-M$.

Through formulae of the Christoffel-Darboux and Liouville-Ostrogradsky type associated with the difference systems (4.5), i.e.: [15]

we get:

$$
\begin{aligned}
& Y_{n+1}^{+}(\bar{x}) Y_{n}(x)-Y_{n}^{+}(\bar{x}) Y_{n+1}(x)=0 \\
& Y_{n+1}^{+}(\bar{x}) Z_{n}(x)-Y_{n}^{+}(\bar{x}) Z_{n+1}(x)=-I
\end{aligned}
$$

i.e., for $x=\bar{x}$ :

$$
Y_{n}^{-1}(x) Z_{n}(x)=\sum_{2=0}^{n-1} Y_{r+1}^{-1}(x)\left(Y_{r}^{+}(x)\right)^{-1}
$$

This shows that the ratio $Y_{n}^{-1}(x) Z_{n}(x)=P_{n}^{-1}(x) Q_{n}(x)$ is negative and monotonically decreasing (as a function of $n$ ) for any $x<-M$.

From the known properties of the matrix orthogonal polynomials one easily obtains, in complete analogy with the scalar case, the decomposition:

$$
P_{n}^{-1}(x) Q_{n}(x)=\sum_{k=1}^{j}\left(x-x_{k}\right)^{-1} y_{k}^{(n)}, \quad n \leqq j \leqq m n
$$

$x_{k}>-M$ being the simple poles of $P_{n}^{-1}(x)$, and $y_{k}^{(n)}$ Hermitian positive semidefinite $m$-matrices. Furthermore, the usual quadrature formula may be easily generalized, i.e., $R_{2 n-1}(x)$ being a matrix polynomial of degree not greater than $2 n-1$ one has:

and this implies:

$$
\left(R_{2 n-1}(x), I\right)=\sum_{k=1}^{j} y_{k}^{(n)} R_{2 n-1}(x)_{k}
$$

$$
S_{p}=\left(I x^{p}, I\right)=\sum_{k=1}^{J} y_{k}^{(n)} x_{k}^{p}, \quad p=0,1, \ldots 2 n-1 .
$$


We can write $P_{n}^{-1} Q_{n}$ in spectral form:

$$
P_{n}^{-1}(x) Q_{n}(x)=\int_{-M}^{\infty}(x-y)^{-1} d T_{n}(y)
$$

with:

$$
T_{n}(-M)=0 ; T_{n}\left(x_{k}+0\right)-T_{n}\left(x_{k}\right)=y_{k}^{(n)}, \quad k=1,2, \ldots j ; T_{n}(x) \text { const }
$$

everywhere else.

An immediate consequence of the decomposition (4.9) is the following expansion of $P_{n}^{-1}(x) Q_{n}(x)$ in descending powers of $x$ :

$$
P_{n}^{-1}(x) Q_{n}(x)=\sum_{k=0}^{2 n-1} S_{k} x^{-k-1}+0\left(x^{-2 n}\right)
$$

and from the representation (4.10) we see that $T_{n}(x)$ is a solution of the finite moment problem:

$$
S_{k}=\int_{-M}^{\infty} x^{k} d T_{n}(x), \quad k=0,1, \ldots 2 n-1 .
$$

The sequence of matrix distribution functions $T_{n}(x)$, with total variation given by $S_{0}=\int_{-M}^{\infty} d T_{n}(x)$, fulfills the conditions of the Helly theorem in matrix form [16]: it exists therefore a matrix distribution function $T_{1}(x)$ and a subsequence $T_{n_{t}}(x)$ such that, in any point of continuity of $T_{1}(x)$ :

$$
\lim _{i \rightarrow \infty} T_{n_{\imath}}(x)=T_{1}(x)
$$

In addition, it is easily seen that $T_{1}(x)$ is a solution of the full matrix moment problem (4.6). If (4.6) is determined, i.e. its solution is unique, we must have $T_{1}(x)=T(x)$.

Taking into account the monotonicity property of $P_{n}^{-1} Q_{n}$ as a function of $n$, when the matrix moment problem is determined we can conclude:

$$
\lim _{n \rightarrow \infty} P_{n}^{-1}(x) Q_{n}(x)=\int_{-M}^{\infty}(x-y)^{-1} d T(y), \quad x<-M .
$$

Furthermore, in complete analogy with the classical theory of Jacobi matrices and continued fractions, it is possible to definite a matrix continued fraction of $J$ type [17], given by the formula:

$$
\begin{gathered}
F_{m}(x)=\left(A_{0} x+B_{0}-\left(A_{1} x+B_{1}-\left(A_{2} x+B_{2}-\cdots\right)^{-1}\right)^{-1}\right)^{-1} \\
=\left(x-A_{0,0}-A_{0,1}\left(x-A_{1,1}-A_{1,2}\left(x-A_{2,2}-\cdots\right)^{-1} A_{2,1}\right)^{-1} A_{1,0}\right)^{-1}
\end{gathered}
$$


It easy to see that its $n$-th order approximants:

$$
\begin{aligned}
F_{m}^{n}(x)= & \left(x-A_{0,0}-A_{0,1}\left(x-A_{1,1}-\cdots\right.\right. \\
& \left.\left.\cdots-A_{n-2, n-1}\left(x-A_{n-1, n-1}\right)^{-1} A_{n-1, n-2}\right)^{-1} \ldots\right)^{-1} A_{1,0}{ }^{-1}
\end{aligned}
$$

coincide with $P_{n}^{-1}(x) Q_{n}(x)$.

Since the $n$-th order approximants to $J$ type continued are also called $[n, n-1]$ Padé approximants, the approximants $P_{n}^{-1} Q_{n}$ are also called $[n, n-1]$ matrix Padé approximants.

\section{Approximation of the Eigenvalue}

Let us apply now the results exposed in the former Section to our case of the moment problems (3.7) and (3.14).

Consider the operator $A$ defined by (3.5) and the two generating basis $g_{i}, i=1, \ldots m$, defined in Section III. As we have seen given the matrix moments: $m_{n}(E, g)=\left(g_{i}, A^{n} g_{k}\right), i, k=1, \ldots m ; n=0,1,2, \ldots$ it is possible to build the sequence of matrix rational functions $P_{n}(-1)^{-1}$ - $Q_{n}(-1)$. This sequence is, in matrix sense, positive and monotonically decreasing for $E<C(g)$, with:

$$
\begin{aligned}
\lim _{n \rightarrow \infty} P_{n}(-1)^{-1} Q_{n}(-1) & =-\int_{-1+\varepsilon}^{\infty}(1+t)^{-1} d T(t) \\
& =-\left(g_{i},(1+A)^{-1} g_{k}\right)=-M_{i, k}
\end{aligned}
$$

and $T(x), M_{i, k}$ as in Section III. Let us consider now the sequence:

$$
f_{n}(E, g)=g^{2}\left\|h^{-1 / 2} V \Omega\right\|^{2}\left(P_{n}^{-1} Q_{n}\right)_{m, m}
$$

i.e. the sequence of the last diagonal elements of the matrices $P_{n}^{-1} Q_{n}$. This last sequence is negative and monotonically decreasing, as it follows from the monotonicity of the matrix sequence. Therefore, if we define $E_{n}(g)$ as the least solution of the equation $E=f_{n}(E, g)$, we get a sequence of solutions $E_{n}(g)$ monotonically decreasing to $E(g)$ as $n \rightarrow \infty$.

This result represents the generalization of that of Ref. [1].

As far as the relationship with the R-S expansion for the ground state eigenvalue is concerned, we have the same result of (1), i.e. the approximants to $E(g)$ require roughly the same information needed to compute the corresponding R-S series coefficients. This fact can be proven by remarking that McClary's argument is still applicable in the present more general case. 
As we have seen in Section IV, the approximants $P_{n}^{-1} Q_{n}$ are uniquely determined by the knowledge of the first $2 n-1$ moments:

$$
\begin{aligned}
P_{n}^{-1} Q_{n} & =\sum_{p=0}^{2 n-1}(-1)^{p+1} m_{p}+0\left(m_{2 n}\right) \\
& =\sum_{p=0}^{2 n-1}(-1)^{p+1}\left(g_{i}, A^{p} g_{k}\right)+0\left(\left(g_{i}, A^{2 n} g_{k}\right)\right) .
\end{aligned}
$$

Let us remember the definition of the generating basis for the field theoretical case:

with:

$$
g_{m}^{-}=c_{m} h^{-1 / 2} V \Omega ; g_{i}=c_{i} h^{-1 / 2} f_{2 i}, \quad i=1, \ldots m-1,
$$

$$
V h^{-1} V \Omega=\sum_{i=1}^{2 m} f_{2 i} ; \quad\left\|g_{i}\right\|=1, \quad i=1, \ldots m .
$$

It is trivially seen that (5.1) holds also in the quantum mechanical case.

Consider any matrix element of the type $\left(g_{i}, A^{n} g_{k}\right), i, k=1, \ldots m$. We have that any matrix element $\left(g_{m}, A^{n} g_{m}\right)$ contains only terms of the type $\left(\Omega, g V h^{-a_{1}} g V \ldots g V h^{-a_{k}} g V \Omega\right)$ with $a_{1}+\cdots+a_{k}=n+1$, and that any element $\left(g_{m}, A^{n} g_{i}\right), i \neq m$, contains only terms of the type

$$
\left(\Omega, g V h^{-a_{1}} g V \ldots g V h^{-a_{k}} f_{2 i}\right), \quad a_{i}+\cdots+a_{k}=n+1
$$

which by (5.1) are in turn contained in the former matrix elements with $a_{1}+\cdots+a_{k+1}=n+2$. The matrix elements $\left(g_{i}, A^{n} g_{j}\right), i, j \neq m$, contain only terms of the type $\left(f_{2 i}, h^{-a_{1}} g V \ldots g V h^{-a_{k}} f_{2 j}\right)$, which are always contained in the terms of the first type with $a_{1}+\cdots+a_{k+2}=n+3$.

Since the terms of the first type with $a_{1}+\cdots+a_{k}=n+1$ are contained in the terms of the R-S expansion up to the $n$-th order [1], it follows that any matrix element $\left(g_{i}, A^{n} g_{j}\right), i, j=1, \ldots m$, is constructed only starting from matrix elements of the R-S expansion up to the order $n+2$.

Consider now in particular the anharmonic oscillators case, in which we know that the orthonormal vectors $e_{n}$ coincide with the harmonic oscillator eigenstates (see Appendix), i.e. $e_{n}=2^{-n}\left(\pi^{1 / 2}(2 n) !\right)^{-1 / 2}$ - $H_{2 n}(x) e^{-1 / 2} x^{2}, n=1, \ldots$. It is thus possible to give the explicit expression for the coefficients $A_{k, k}$ and $A_{k, k+1}$ of the matrix continued fraction (4.10), whose approximants are $P_{k}^{-1} Q_{k}$. By (3.11) and (3.12) we have:

$$
\begin{aligned}
& A_{k, k}=\left(\begin{array}{ccc}
a_{k m+1, k m+1} & \ldots & a_{k m+1, k m+m} \\
\vdots & \vdots & \vdots \\
a_{k m+m, k m+1} & \ldots & a_{k m+m, k m+m}
\end{array}\right), \\
& A_{k, k+1}=\left(\begin{array}{ccccccccc}
b_{k m+1} & 0 & 0 & 0 & 0 & 0 & 0 & 0 & 0 \\
\ddots & & & & & & & & \\
& \ddots & \ddots & & & & & & \\
a_{k m+m, k m+m+1} & & b_{k m+m}
\end{array}\right),
\end{aligned}
$$


and any easy computation yields (remember that $V=2^{-m}((2 m-1) !)^{-1 / 2}$ $\cdot H_{2 m}(x)$

$$
\begin{gathered}
a_{k, k}=\left(e_{k}, A e_{k}\right)=g \frac{\sqrt{2 m(2 m) !}}{2 k}\left(\begin{array}{c}
(2 k) \\
m
\end{array}\right)-\frac{E}{2 k}, \\
b_{k}=\left(e_{k}, A e_{k+m}\right)=\frac{g}{2} \frac{\sqrt{2 m(2 m) !}}{\sqrt{k(k+m)}} \sqrt{\left(\begin{array}{c}
2 k+2 m) \\
2 m
\end{array}\right),} \\
a_{k, k+j}=\frac{g}{2} \sqrt{\frac{2 m(2 m) !}{k(k+j)}} \sqrt{\left(\begin{array}{c}
2 k \\
m-j
\end{array}\right)\left(\begin{array}{c}
2 k+2 j \\
m+j
\end{array}\right)}, \quad j=1, \ldots m-1 .
\end{gathered}
$$

\section{Appendix}

Let $A$ be a self-adjoint operator in a Hilbert space $H$ and let the $m$ non-zero orthogonal vectors $g_{1}, \ldots g_{m}$ belong to $C^{\infty}(A)$. Consider the sequence of vectors $A^{n} g_{i}, i=1, \ldots m ; n=0,1,2 \ldots$, which will be assumed to be linearly independent. Through an orthogonalization procedure of the Gram-Schmidt type, it is possible to obtain from the above sequence a system of orthonormal vectors satisfying. a $2 m+1$-term recurrence relation.

Putting indeed $g_{i}=f_{i}, i=1, \ldots m$, let us define:

$$
\begin{gathered}
f_{m+1}=\left(A-D_{1,1}\right) f_{1}-D_{1,2} f_{2}-\cdots-D_{1, m} f_{m} \\
f_{m+2}=-D_{2,1} f_{1}+\left(A-D_{2,2}\right) f_{2}-\cdots-D_{2, m+1} f_{m+1} \\
f_{2 m-1}=-D_{m-1,1} f_{1}-\cdots-\left(A-D_{m-1, m-1}\right) f_{m-1}-D_{m-1,2 m} f_{2 m-2} \\
f_{k+m}=-W_{k}^{2} W_{k-m}^{-2} f_{k-m}-D_{k, k-m+1} f_{k-m+1}+\cdots \\
\cdots+\left(A-D_{k, k}\right) f_{k}-\cdots-D_{k, k+m-1} f_{k+m-1} \\
k=m, m+1, m+2, \ldots
\end{gathered}
$$

where:

$$
W_{i}^{2}=\left(f_{i}, f_{i}\right) ; D_{i, j}=\left(f_{i}, A f_{j}\right) W_{j}^{-2} .
$$

It is easy to check the orthogonality $\left(f_{i}, f_{k}\right)=0, i \neq k$.

Defining $e_{k}=W_{k}^{-1} f_{k}$, we get a sequence of orthonormal vectors $e_{k}$ satisfying the following $2 m+1$-term recurrence relation:

$$
A e_{k}=b_{k-m} e_{k-m}+a_{k, k-m+1} e_{k-m+1}+\cdots+a_{k, k} e_{k}+\cdots+b_{k} e_{k+m}
$$

where:

$$
\begin{gathered}
b_{k-m}=W_{k} W_{k-m}^{-1}, \quad a_{i, j}=W_{i}^{-1} W_{j}^{-1}\left(f_{i}, A f_{j}\right) \\
a_{i, j}=0 ; i, j \leqq 0 ; \quad b_{i}=0 ; i \leqq 0 .
\end{gathered}
$$


Consider in particular the anharmonic oscillator case: let $g_{1}=c_{1} H_{2}(x) e^{-x^{2} / 2}, \ldots g_{m}=c_{m} H_{2 m} e^{-x^{2} / 2}, c_{i}$ being normalization constants, and let the operator $A$ be defined by (3.5).

Since we have

$$
A H_{2 i}(x) e^{-x^{2} / 2}=\sum_{j=\max (i-m, 1)}^{i+m} d_{j} H_{2 j}(x) e^{-x^{2} / 2}
$$

it is easily seen that the above orthogonalization procedure applied to the vectors $A^{n} g_{i}, i=1, \ldots m ; n=0,1, \ldots$ yields the harmonic oscillator eigenstates, i.e.

$$
e_{m}=\frac{H_{2 m} e^{-x^{2} / 2}}{2^{m} \sqrt{\pi^{1 / 2}(2 m) !}}, \quad m=1,2, \ldots .
$$

Consider now, in general, the orthonormal vectors $e_{k}$ obtained through the above described orthogonalization procedure. If we arrange them into $m$-component column vectors

$$
E_{k}=\left(\begin{array}{c}
e_{k m+1} \\
\vdots \\
e_{k m+m}
\end{array}\right), \quad k=0,1,2, \ldots
$$

we get a three-term $m \times m$ matrix recurrence relation:

$$
A E_{k}=A_{k, \bar{k}-1} E_{k-1}+A_{k, k} E_{k}+A_{k, k+1} E_{k+1}
$$

where:

$$
\begin{aligned}
& A_{k, k}=A_{k, k}^{+}=\left(\begin{array}{cccc}
a_{k m+1, k m+1} & \ldots & a_{k m+1, k m+m} \\
a_{k m+m, k m+1} & \ldots & a_{k m+m, k m+m}
\end{array}\right),
\end{aligned}
$$

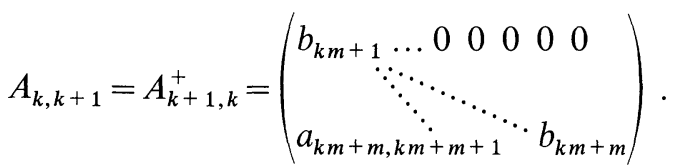

Given now any two $m$-component vectors $F, G$, let us define as their "scalar product" the following $m$-matrix:

$$
(F, G)=\left(\left(f_{i}, g_{j}\right)\right), \quad i, j=1, \ldots m
$$

where $\left(f_{i}, g_{j}\right)$ is the scalar product between $f_{i}$ and $g_{j}$ in $H$.

In this notation, the matrix moments $S_{n}=\left(e_{i}, A^{n} e_{j}\right), i, j=1, \ldots m$ take the simple form:

$$
S_{n}=\left(E_{0}, A^{n} E_{0}\right) \text {. }
$$


It is straightforward to check that this matrix "scalar product" satisfies properties analogous to those of the usual one, i.e.:

a) $(F, G)=(G, F)^{+}$.

b) $(F, F)$ is a positive semidefinite matrix; in addition $(F, F)=0$ if and only if $F \equiv 0$, and its rank is $m$ if and only if the components of $F$ are linearly independent.

c) $C_{1}$ and $C_{2}$ being arbitrary constant matrices, one has:

$$
\left(C_{1} F_{1}+C_{2} F_{2}, G\right)=C_{1}\left(F_{1}, G\right)+C_{2}\left(F_{2}, G\right) .
$$

a) and c) yield:

$$
\left(F, C_{1} G_{1}+C_{2} G_{2}\right)=\left(F, G_{1}\right) C_{1}^{+}+\left(F, G_{2}\right) C_{2}^{+} .
$$

Of course $F$ and $G$ will be orthonormal with respect to the "scalar product" (A.1) if and only if their components are orthonormal with respect to the scalar product in $H$. It follows that the set of $m$-vectors $E_{k}$, $k=0,1,2, \ldots$ is orthonormal with respect to (A.1), i.e. $\left(E_{i}, E_{k}\right)=\delta_{i k} I$.

Consider now the $m$-vectors

$$
R=P(A) E_{0}=\sum_{k=0}^{n} C_{k} A^{k} E_{0}, \quad S=Q(A) E_{0}=\sum_{k=0}^{p} D_{k} A^{k} E_{0},
$$

where $P(A)$ and $Q(A)$ are matrix polynomials in $A$.

Their matrix "scalar product" is:

$$
(R, S)=\left(P(A) E_{0}, Q(A) E_{0}\right)=\sum_{k=0}^{n} \sum_{j=0}^{p} C_{k} S_{k+J} D_{j}^{+}=\{P(x), Q(x)\},
$$

where use of (A.2) has been made, and $\{P(x), Q(x)\}$ is the matrix "scalar product" defined in Section 4 in the space of the $m$-matrices.

This shows that the "scalar product" (in the sense of this appendix) between the above vectors coincides with the one defined in the space of the matrix polynomials. We can thus write: $E_{k}=P_{k}(A) E_{0}$, and these vectors satisfy, if we replace $X$ with $A$, the same recurrence relations (4.3) valid for $P_{k}(x)$, where the $P_{k}(x)$ are the matrix polynomials orthonormal with respect to the matrix distribution function $T(x)$.

Acknowledgments. It is a pleasure to thank Professors G. Velo and B. Simon for their interest in this work and for their constructive criticism.

\section{References}

1. McClary, W. K.: Commun. math. Phys. 24, 171 (1972)

2. See, e.g.: Graffi, S., Grecchi, V., Turchetti, G.: Nuovo Cimento 4B, 313 (1971)

3. Simon, B.: Phys. Rev. Letters 25, 1583 (1970)

4. Graffi, S., Grecchi, V., Simon, B.: Phys. Letters 32 B, 631 (1970) 
5. Loeffel, J.J., Martin, A., Simon, B., Wightman, A.S.: Phys. Letters 30B, 656 (1969)

6. Nussbaum, A.E.: Studia Math. 33, 305 (1969)

Masson, D., McClary, W. K.: J. Funct. Anal. 10, 19 (1972)

Simon, B.: Indiana Univ. Math. J. 20, 1145 (1971)

7. Akhiezer, N. I.: The Classical Moment Problem. Edinburgh-London: Oliver \& Boyd 1965

8. Achieser, N.I., Glasmann,I.M.: Theorie der linearen Operatoren in Hilbert Raum. Berlin: Akademie-Verlag 1954, Chapter VI

9. Glimm,J., Jaffe, A.M.: Phys. Rev. 176, 1945 (1968); Ann. Math. 91, 362 (1970); Acta Math. 125, 203 (1970)

10. Simon, B., Hoegh-Krohn, R.: J. Funct. Anal. 9, 121 (1972)

11. Simon, B.: Ann. Phys. 58, 76 (1970)

12. Hellwig, G.: Differentialoperatoren der mathematischen Physik, p. 159. BerlinGöttingen-Heidelberg: Springer 1959

13. See, e.g. Naimark, M. A.: Linear Differential Operators, Part II. London: Harrap 1968

14. Krein, M. G.: Dokl. Akad. Nauk SSSR 69, 125 (1949)

15. Atkinson,F.V.: Discrete and Continuous Boundary Value Problems. New YorkLondon: Academic Press 1964, Chapter VI

16. Ref. [15], Appendix 18

17. MacNerney, J.S.: Duke Math. J. 26, 663 (1959)

Communicated by A.S. Wightman

\author{
S. Graffi \\ V. Grecchi \\ Istituto Nazionale di Fisica Nucleare, \\ Sezione di Bologna \\ Bologna, Italy
}

